\title{
Editorial
}

\section{Development of multimodal neuroimaging markers for neurological disorders - Part 1}

\author{
Kelvin K.L. Wong ${ }^{\mathrm{a}, *}$, Defeng Wang ${ }^{\mathrm{b}}$ and Peipeng Liang ${ }^{\mathrm{c}}$ \\ ${ }^{a}$ School of Medicine, Western Sydney University, Sydney, New South Wales, Australia \\ ${ }^{\mathrm{b}}$ Department of Imaging and Interventional Radiology, Research Center for Medical image Computing, \\ The Chinese University of Hong Kong, Hong Kong \\ ${ }^{\mathrm{c}}$ Department of Radiology, Xuanwu Hospital, Capital Medical University, Beijing, China
}

Multi-modal neuroimaging, including electroencephalography (EEG), magnetoencephalography (MEG), positron emission tomography (PET), magnetic resonance imaging (MRI), Near-infrared spectroscopy (NIRS), and single-photon emission computed tomography (SPECT), have been widely used in the diagnosis of neurological diseases, such as Alzheimer's disease (AD), Parkinson's disease (PD), and multiple sclerosis, etc. Each neuroimaging technique has its own merits, and multi-modal neuroimaging is helpful for further improving the diagnosis accuracy of neurological diseases.

This special issue focuses on the development of neuroimaging markers for early diagnosis of neurological disorders. The goal is to provide an overview to the current state-of-the-art advances in disease diagnosis using multi-modal neuroimaging techniques and promote further discussion of applying the imaging technologies in future clinical medicine. This special issue includes 10 research articles that cover a wide range of topics related to recent advances in neuroimaging marker studies of neurological disease, which can be the development and evaluation of new methods for neuroimaging data analysis and their applications. The applications include: combining clinical measures, behavioral performance with neuroimaging data to improve disease screening, diagnosis, progress monitoring, as well as predicting disease prognosis and treatment efficacy.

The first part of this special issue includes 5 articles. The first two articles examine the disease-related alterations in resting state of human brain and the next three articles focus on the clinical diagnosis and applications of Electrocardiograph (ECG), digital subtraction angiography (DSA) and computed tomography (CT) in neurological disease. Brief discussion of these studies are as follows.

Resting-state fMRI is a functional brain imaging method, which can be used to evaluate regional interactions that occur when a subject is not performing an explicit task [1]. The first two papers address the resting-state fMRI as below. Tan, et al, explored the alterations of regional synchronization in infantile spasm patients during resting state through regional homogeneity analysis [2]. Although

\footnotetext{
*Corresponding author: Kelvin Kian Loong Wong, School of Medicine, Building 30, Western Sydney University Campbelltown Campus, Narellan Rd \& Gilchrist Dr, Campbelltown NSW 2560, Australia. Tel.: +61 4620 3620; E-mail: kelvin. wong@westernsydney.edu.au.
} 
the semiology and brain activity of epilepsy has been widely studied in adults, relatively few studies have examined in infants and children. Previous imaging studies mainly focus on the domain of childhood absence [3] and chronic benign epilepsy [4]. Only one study using the MRI approach examined the altered structural connectivity of infantile spasm patients [5]. This is the first study to explore the altered brain functional connectivity of infantile spasm patients. It investigated the functional modulations in the resting state in the infantile spasm patients and found that, some regions showed significant relationship with the epilepsy duration in the patients. The study provided useful experimental results, which may enable researchers to detect the core region of infantile spasm epilepsy, as well as help clinicians better identify disease type, perform more accurate clinical assessment, and disease treatment.

Liu, et al, assessed the acupuncture effect on the human brain [6]. Although acupuncture has long been used to treat various diseases, a full understanding of the method has not been yet acheived [7]. The application of fMRI has provided practical methods to explore the mechanisms of acupuncture treatment and visually identify connections within the brain [8]. Liu et al. applied fMRI to observe the cerebral activation effects of acupuncture at the Zusanli and Yanglingquan points. The results indicated that, stimulating a combination of acupoints was also able to activate new brain areas compared to each single acupoint, which suggests the ability to generate new curative effects [9]. The contribution of this article lies on the new findings that may aid healthcare providers who wish to evaluate and integrate traditional medicine into mainstream practice, which can provide supplementary method leading to improve patient care.

Generally, the Electrocardiograph (ECG), digital subtraction angiography (DSA), and computed tomographic (CT) are the common tools used in the current clinical practice to detect and diagnose neurological diseases. Three articles reported new studies in this field. Gong et al. examined the potential of heart rate variability (HRV), which is a 2D neuroimaging signal, as an efficient tool for predicting the onset of epilepsy in children [10]. Although there were many studies regarding the relation between HRV and adult with epilepsy [11, 12], the relation between the HRV and the children with epilepsy are seldom explored. The results from this study demonstrated the changes of HRV for the children with refractory epilepsy. The HRV parameters were divided into three different segments. It was found that the values of mean heart rate (meanHR), low frequency (LF), and LF/ high frequency (HF) were significantly greater in the third segment. Additionally, the values of mean inter-beat rate (meanRR) and HF were significantly less in this segment. The study results indicated that the sympathetic nervous system become more active at onset period. Thus, the study concluded that, the parameters including meanHR, meanRR, LF, HF, and LF/HF may potentially be the useful clinical indices to predict seizures.

Chen et al. investigated the basal vein in posterior incisural space, and performed anatomical comparison based on autopsy, venous phases of DSA and CTV [13]. Given the narrowness of the posterior incisural space, the basal vein in this space is susceptible to be obstructed during surgical procedures, and venous infarction can occur along with venous damage [14]. The preoperative medical diagnosis on the cerebral venous system in neurological diseases is of crucial importance in planning an intracranial surgery [15]. This study compared to the cadavers, in the patients, DSA and CTV revealed 90\% and $95 \%$ of the basal vein, respectively. The types and variation of basal veins were identified in both cadavers, DSA, and CTV. The study showed that preoperative image-based examinations were helpful in the design of individualized surgical approaches in the posterior incisural space. This study provides important information with respect to precluding the basal vein damage and reducing postoperative complications.

Wang et al. investigated distinctive patterns of functional connectivity in each subregion of amygdala in $\mathrm{AD}$ [16]. Resting-state fMRI is a promising technique for measuring spontaneous brain activity. By using the fMRI method, this study focused on amygdale segmentation and proposed its accu- 
rate functional disconnection pattern in the $\mathrm{AD}$ patients. Recent researches on $\mathrm{AD}$ have shown that altered structure and function of amygdale, which provided a promising indicator of AD [17]. However, previous studies often considered the amygdale as a single structure [18], and little is known about the functional connectivity changes of amygdale subregions in AD subjects. In this study, three subregions of amygdale were defined according to probabilistic cytoarchitectonic atlases and the wholebrain resting-state functional connectivity was then calculated for each subregion. By comparing with controls, the distinct disruption patterns of amygdale subregional functional connectivity in the AD patients were found. These findings have important implications for the underlying neurobiology of $\mathrm{AD}$ and add the new evidence for the disconnection syndrome of $\mathrm{AD}$, which may provide the potential biomarker for detecting early $\mathrm{AD}$ in the future.

\section{References}

[1] B.B. Biswal, Resting state fMRI: A personal history, Neuroimage 62(2) (2012), 938-944.

[2] Z. Tan, Y. Li, D. Zang, H. Zhang, C. Zhao, H. Jiang, Y. Chen, D. Cao, L. Chen, J. Liao, Q. Chen and G. Luan, Altered regional homogeneity in epileptic patients with infantile spasm: A resting-state fMRI study, J Xray Sci Technol $\mathbf{2 4}$ (2016), 285-295.

[3] T. Yang, et al., Altered spontaneous activity in treatment-naive childhood absence epilepsy revealed by regional homogeneity, Journal of the Neurological Sciences 340(1) (2014), 58-62.

[4] H. Zeng, et al., Regional homogeneity (ReHo) changes in new onset versus chronic benign epilepsy of childhood with centrotemporal spikes (BECTS): A resting state fMRI study, Epilepsy Research 116 (2015), 79-85.

[5] C. Clark, et al., Quantitative MRI evidence for altered structural remodelling of the temporal lobe in cryptogenic West syndrome, Epilsepsia 56(4) (2015), 608-616.

[6] L. Liu, Y. Wu, J. Zheng, X. Lai, D. Zeng, H. Li, C. Shi, M. Yang and C. Liang, Cerebral activation effects of acupuncture using Zusanli (ST36) and Yanglingquan (GB34) points based on Regional Homogeneity indices: A resting-state fMRI study, J Xray Sci Technol 24 (2016), 297-308.

[7] S. Zhou, F. Zeng, J. Liu, et al., Influence of acupuncture stimulation on cerebral network in functional diarrhea, Evidence-Based Complementary and Alternative Medicine, vol. 2013, Article ID 975769, 9 pages, 2013. doi:10.1155/2013/975769

[8] C.A. Brown and A.K. Jones, A role for midcingulate cortex in the interruptive effects of pain anticipation on attention, Clinical Neurophysiology 119(10) (2008), 2370-2379.

[9] J. Shen, Research on the neurophysiological mechanisms of acupuncture: Review of selected studies and methodological issues, The Journal of Alternative \& Complementary Medicine 7(1) (2001), 121-127.

[10] X. Gong, X. Mao, Y. Chen, L. Huang, W. Liu, X. Huang, Z. Tan, X. Wang, H. Zhang, W. Wu, Q. Chen and R. Li, The changes of HRV in refractory epilepsy: The potential index to predict the onset of epilepsy in children. $J$ Xray Sci Technol 24 (2016), 309-317.

[11] F. Leutmezer, et al., Electrocardiographic changes at the onset of epileptic seizures. Epilepsia 44(3) (2003), 348-354.

[12] R.A. Sarkis, et al., Autonomic changes following generalized tonic clonic seizures: An analysis of adult and pediatric patients with epilepsy, Epilepsy Research 115 (2015), 113-118.

[13] D. Chen, et al., Basal vein in the posterior incisural space: An anatomical comparison based on autopsy, digital subtraction angiography and computed tomographic venography, J Xray Sci Technol 24 (2016), 319-327.

[14] A. Ardeshiri, et al., Microsurgical anatomy of the mesencephalic veins, Journal of Neurosurgery 106(5) (2007), 894-899.

[15] H. Han, et al., Dural entrance of the bridging vein in the middle cranial fossa: A novel classification of the cerebral veins for preoperative planning, Neurosurgery 67(3) (2010), ons9-ons15.

[16] Z. Wang, M. Zhang, Y. Han, H. Song, R. Guo and K. Li, Differentially disrupted functional connectivity of the subregions of the amygdala in Alzheimer's disease, J Xray Sci Technol 24 (2016), 329-342.

[17] M.I. Miller, et al., Amygdalar atrophy in symptomatic Alzheimer's disease based on diffeomorphometry: The BIOCARD cohort, Neurobiology of Aging 36 (2015), S3-S10.

[18] H. Yao, et al., Decreased functional connectivity of the amygdala in Alzheimer's disease revealed by resting-state fMRI, European journal of radiology 82(9) (2013), 1531-1538. 